\title{
Direct evidence of heteroleptic complexation in the macroscopic dynamics of metallo-supramolecular polymer networks
}

\author{
Mostafa Ahmadi, Sebastian Seiffert*
}

Department of Chemistry, Johannes Gutenberg-Universität Mainz, Duesbergweg 10-14, D-55128 Mainz, Germany

${ }^{*}$ Correspondence to Sebastian Seiffert (sebastian.seiffert@uni-mainz.de)

\section{- Supporting Information -}

Following the time-temperature superposition (tTS) rule, shear stress curves obtained at different temperatures are horizontally shifted to overlay and form a master-curve at $25^{\circ} \mathrm{C}$, as shown in Figure 3 of the main manuscript. The activation energies $\left(E_{\mathrm{a}}\right)$ for dissociation of metal-ligand complexes sre calculated by considering an Arrhenius-type temperature dependence for the horizontal shift factor $\left(a_{T}\right)$ :

$a_{T}=\exp \left[\left(\frac{E_{a}}{R}\right)\left(\frac{1}{T}-\frac{1}{T_{0}}\right)\right]$

where $R$ is the gas constant and $T$ is the absolute temperature. The subscript " 0 " refers to the reference temperature of $25^{\circ} \mathrm{C}$.

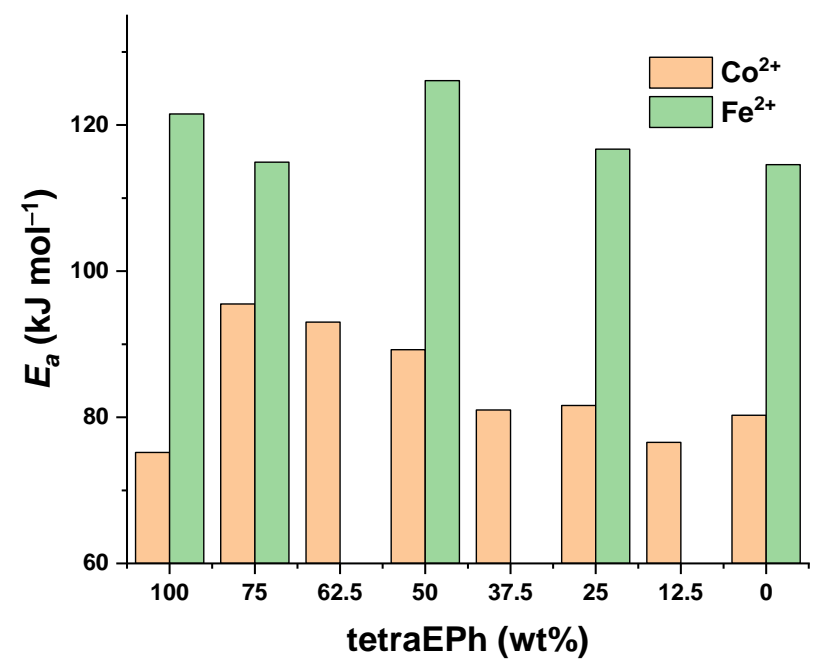

Figure S1. Dissociation activation energies of transient networks formed by tetraMix $(\mathrm{X}) \mathrm{M}(1)$ at different network compositions, calculated from time-Temperature superposition of shear stress curves obtained at different temperatures. 


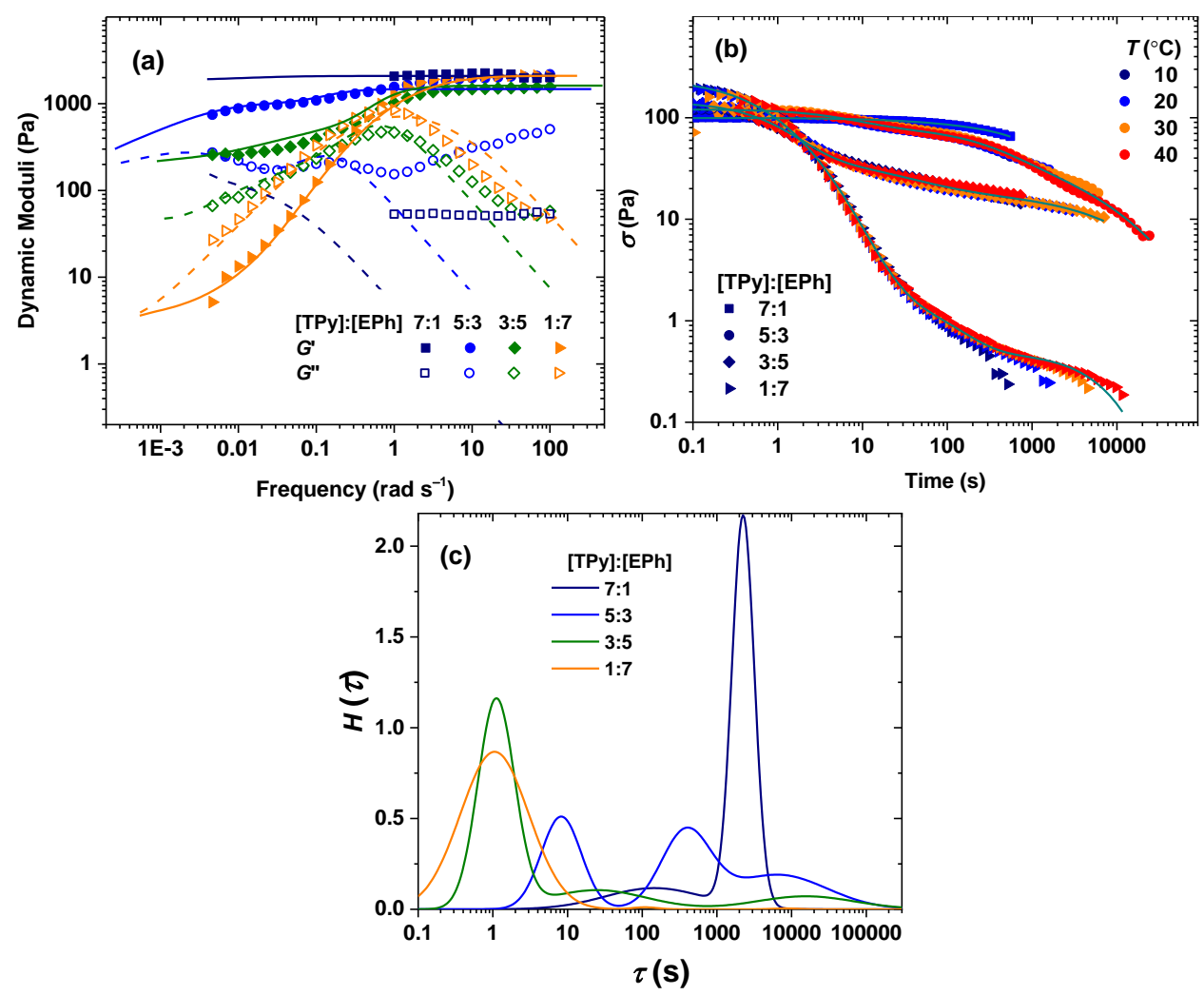

Figure S2. (a) Dynamic storage (filled symbol and solid lines) and loss (open symbols and dashed lines) moduli curves, (b) shear stress master-curves of transient networks at the reference temperatures of $25{ }^{\circ} \mathrm{C}$, and (c) relaxation time spectra of transient networks at the reference temperatures of $25{ }^{\circ} \mathrm{C}$, obtained by tetraMix $(\mathrm{X}) \mathrm{Co}(1)$ at $\varphi=60 \mathrm{~g} \mathrm{~L}^{-1}$ and different network compositions designated in legends. Solid shear stress lines are fitted curves obtained using up to three relaxation modes. Dynamic moduli lines are accordingly obtained by transforming step-strain fits to dynamic moduli. 


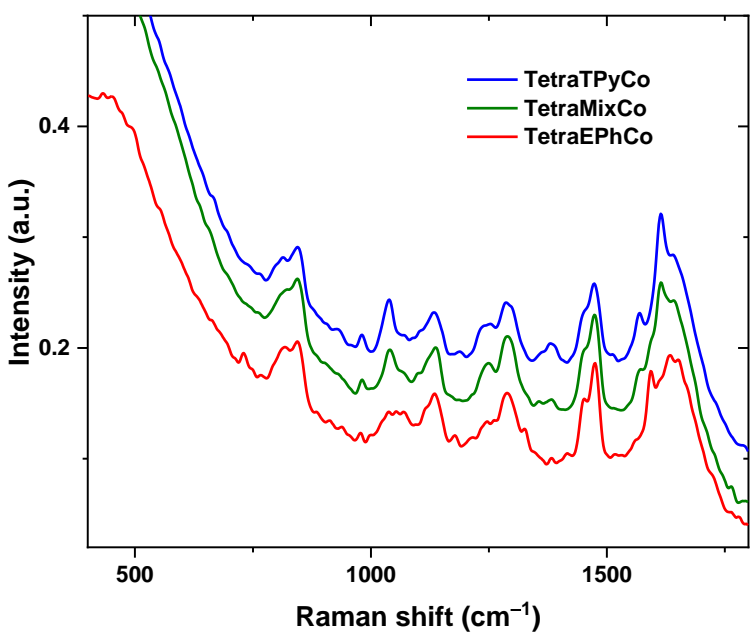

Figure S3. Raman spectra of parent networks and their equimolar mixture in the presence of $\mathrm{Co}^{2+}$ at the stoichiometric metal to ligand ratio.

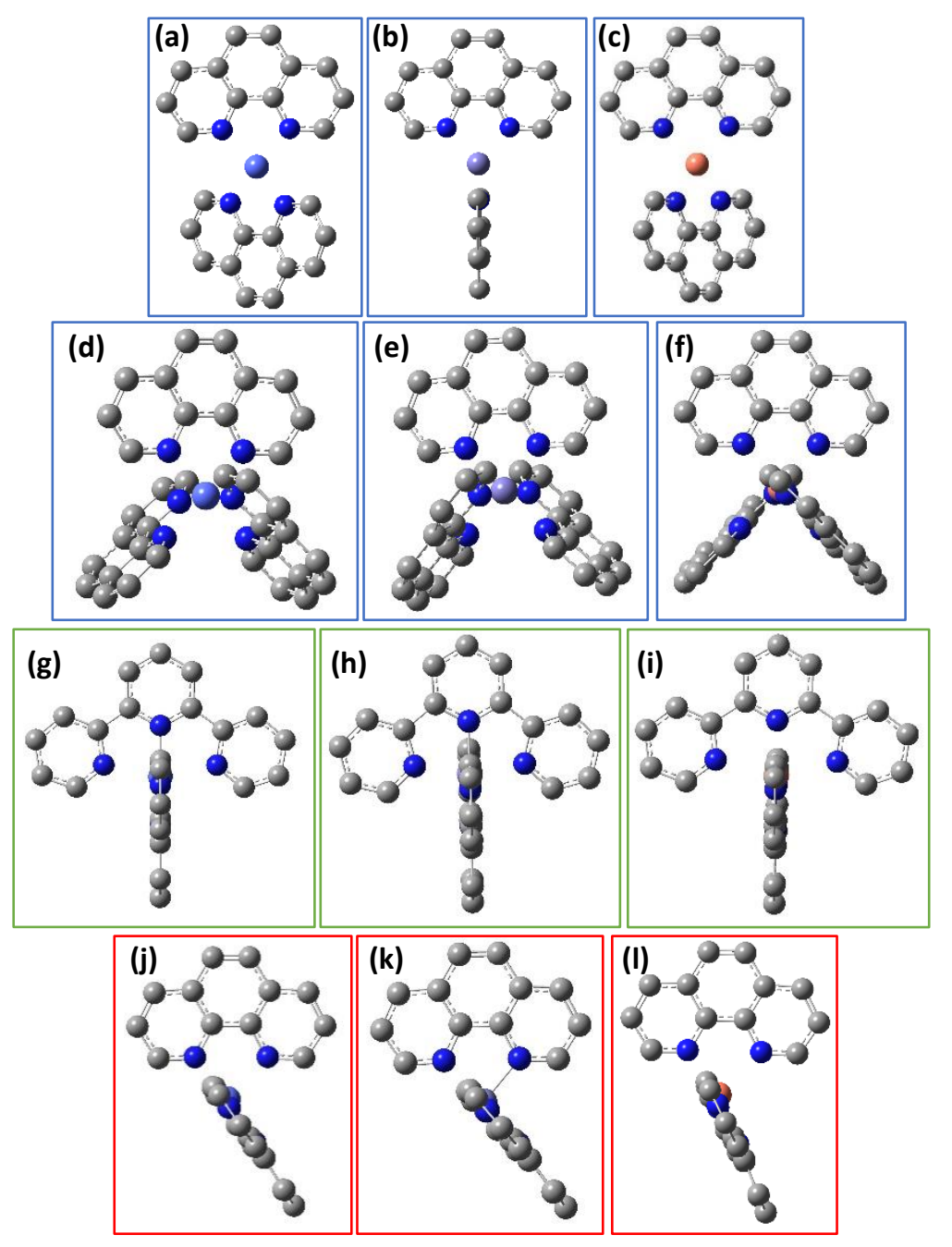


Figure S4. Optimized structures of (a) Co-BisPhen , (b) Fe-BisPhen, (c) Cu-BisPhen, (d) Co-TrisPhen, (e) FeTrisPhen, (f) Cu-TrisPhen, (g) Co-BisTPy , (h) Fe-BisTPy, (i) Cu-BisTPy, (j) Co-Heteroleptic, (k) Fe-Heteroleptic, (I) $\mathrm{Cu}$-Heteroleptic.

Table S1. Bond length (Å) between nitrogen atoms of ligands and coordinated metal ions. Three bond lengths of Tris-complexes designate those of the symmetric ligand and the short and long bond lengths of the asymmetric two other ligands. Four bond lengths of the Heteroleptic complex designate those of the asymmetric terpyridine and the asymmetric phenanthroline ligands. Angles between two ligands are shown for the asymmetric complexes in the last column.

\begin{tabular}{|l|l|l|l|l|l|l|}
\hline Ion & Complex & $\mathbf{L}_{\mathbf{1}}{ }^{\mathbf{a}}$ & $\mathbf{L}_{\mathbf{1}}$ or $\mathbf{L}_{\mathbf{2}}$ & $\mathbf{L}_{\mathbf{2}}$ & $\mathbf{L}_{\mathbf{2}}$ & Angle \\
\hline $\mathbf{C o}$ & MonoPhen & 1.896 & & & & \\
\hline $\mathbf{C o}$ & BisPhen & 1.979 & & & & 150.89 \\
\hline $\mathbf{C o}$ & TrisPhen & 2.008 & 2.252 & 2.011 & & \\
\hline $\mathbf{C o}$ & MonoTPy & 1.876 & & & & \\
\hline $\mathbf{C o}$ & BisTPy & 1.893 & 2.030 & & & \\
\hline $\mathbf{C o}$ & Heteroleptic & 1.891 & 1.996 & 1.976 & 2.148 & 123.6 \\
\hline $\mathbf{F e}$ & MonoPhen & 1.917 & & & & \\
\hline $\mathbf{F e}$ & BisPhen & 1.985 & & & & 90 \\
\hline $\mathbf{F e}$ & TrisPhen & 2.024 & 2.028 & 2.019 & & \\
\hline $\mathbf{F e}$ & MonoTPy & 1.921 & & & & \\
\hline $\mathbf{F e}$ & BisTPy & 1.917 & 2.024 & & & \\
\hline $\mathbf{F e}$ & Heteroleptic & 1.918 & 2.016 & 1.960 & 2.002 & 128.11 \\
\hline $\mathbf{C u}$ & MonoPhen & 1.935 & & & & \\
\hline $\mathbf{C u}$ & BusPhen & 2.015 & & & & \\
\hline
\end{tabular}

${ }^{a}$ : $L_{1}$ and $L_{2}$ can be Phen or TPy ligands depending on the complex. Bond lengths of Phen nitrogen atoms are highlighted in red and those of TPy nitrogen atoms are shown in black. 
Table S2. Sum of electronic and thermal free energies of the optimized structures.

\begin{tabular}{|c|c|c|c|}
\hline$\Delta G\left(\mathrm{kcal} \mathrm{mol}^{-1}\right)$ & Co & Fe & $\mathrm{Cu}$ \\
\hline MonoPhen & -286.15 & -307.98 & -302.65 \\
\hline BisPhen & -111.88 & -124.53 & -96.36 \\
\hline TrisPhen & -389.79 & -336.76 & -338.24 \\
\hline MonoTPy & 38.13 & 81.83 & 59.73 \\
\hline BisTPy & -542.98 & -824.64 & -768.39 \\
\hline Heteroleptic1 $^{\text {a }}$ & -178.80 & -161.63 & -360.87 \\
\hline${\text { Heteroleptic } 2^{b}}^{b}$ & -503.08 & -551.45 & -723.24 \\
\hline
\end{tabular}

a: Phenanthroline associates first, ${ }^{\text {b: }}$ Terpyridine associates first.
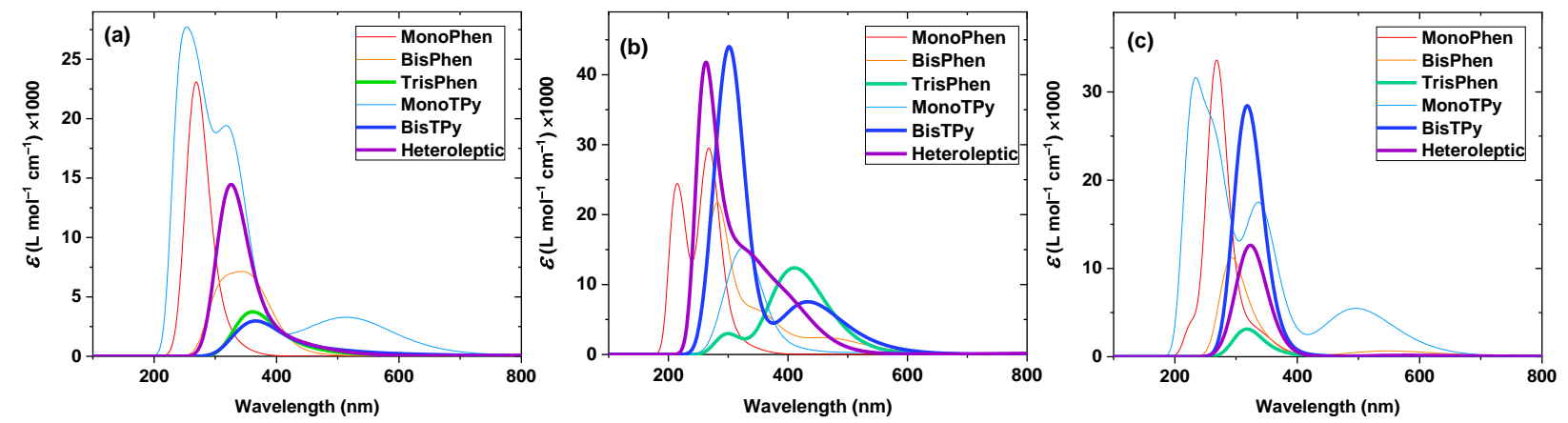

Figure S5. Molar extinction coefficients of different metal-ligand complexes made by (a) $\mathrm{Co}^{2+}$, (b) $\mathrm{Fe}^{2+}$, and (c) $\mathrm{Cu}^{2+}$ metal ions. The specific absorption bands of heteroleptic complexes overlay those of the homoleptic complexes, completely when $\mathrm{Co}^{2+}$ or $\mathrm{Cu}^{2+}$ is employed, and partially when $\mathrm{Fe}^{2+}$ is utilized. 

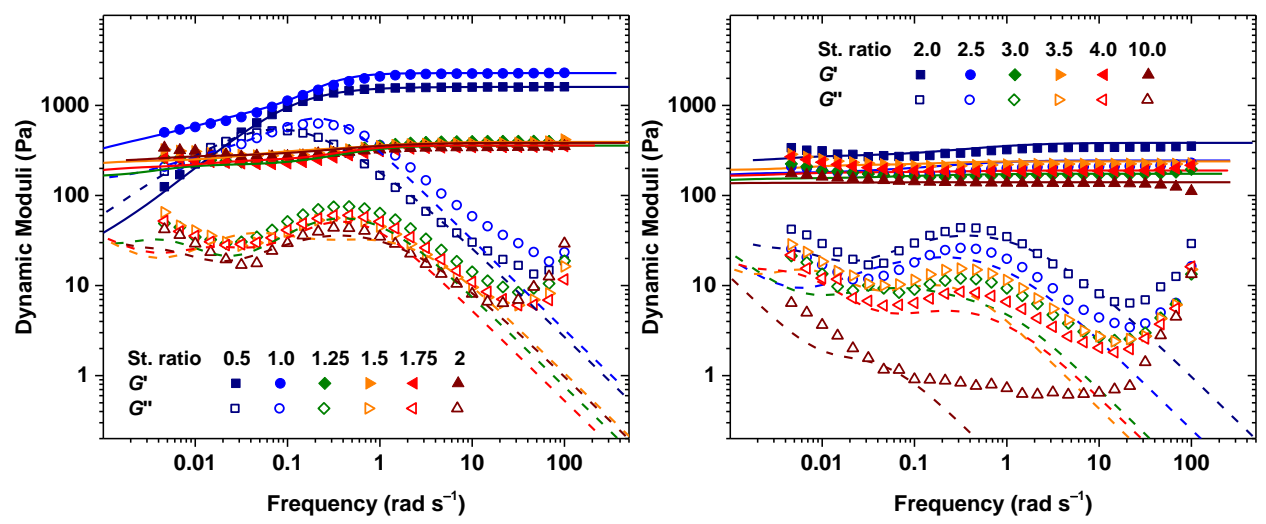

Figure S6. Dynamic storage (filled symbol and solid lines) and loss (open symbols and dashed lines) moduli curves of transient networks obtained by tetraMix $(0.5) \operatorname{Co}(X)$ at $\varphi=60 \mathrm{~g} \mathrm{~L}^{-1}$ and metal to ligand stoichiometric ratios designated in legends. Symbols are obtained by oscillatory shear measurements and lines are obtained by transforming step-strain fits to dynamic moduli.
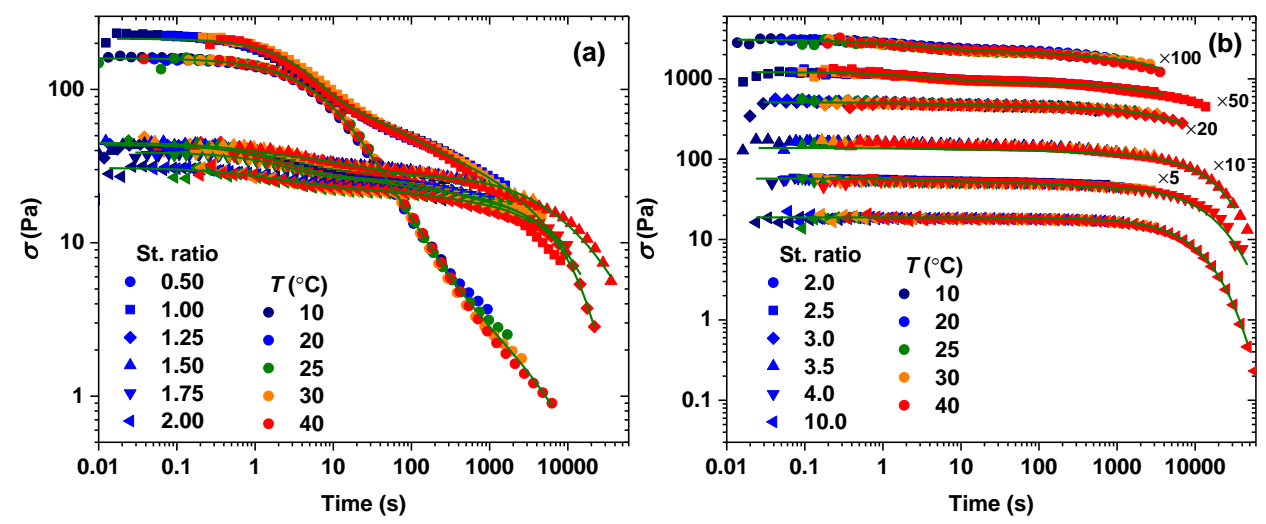

Figure S7. Shear stress master-curves of transient networks obtained at the reference temperatures of $25^{\circ} \mathrm{C}$ by tetraMix $(0.5) \operatorname{Co}(\mathrm{X})$ at $\varphi=60 \mathrm{~g} \mathrm{~L}^{-1}$ and metal to ligand stoichiometric ratios designated in legends. Solid lines are fitted curves obtained considering up to three relaxation modes. 

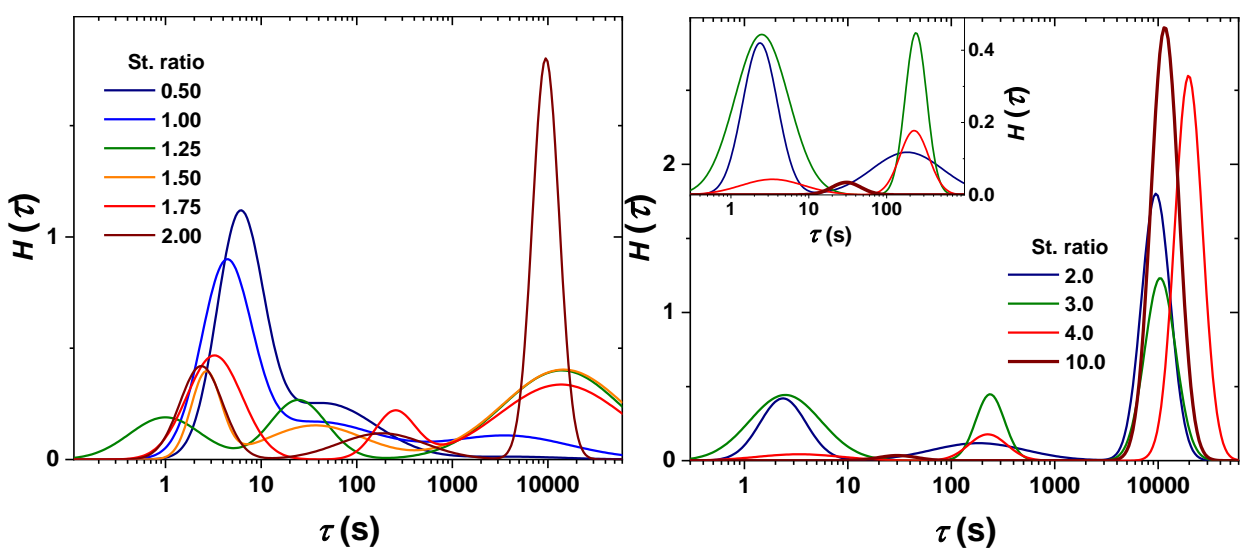

Figure S8. Relaxation time spectra of transient networks obtained by tetraMix(0.5) $\mathrm{Co}(\mathrm{X})$ at $\varphi=60 \mathrm{~g} \mathrm{~L}^{-1}$ and metal to ligand stoichiometric ratios designated in legends, at the reference temperatures of $25^{\circ} \mathrm{C}$.
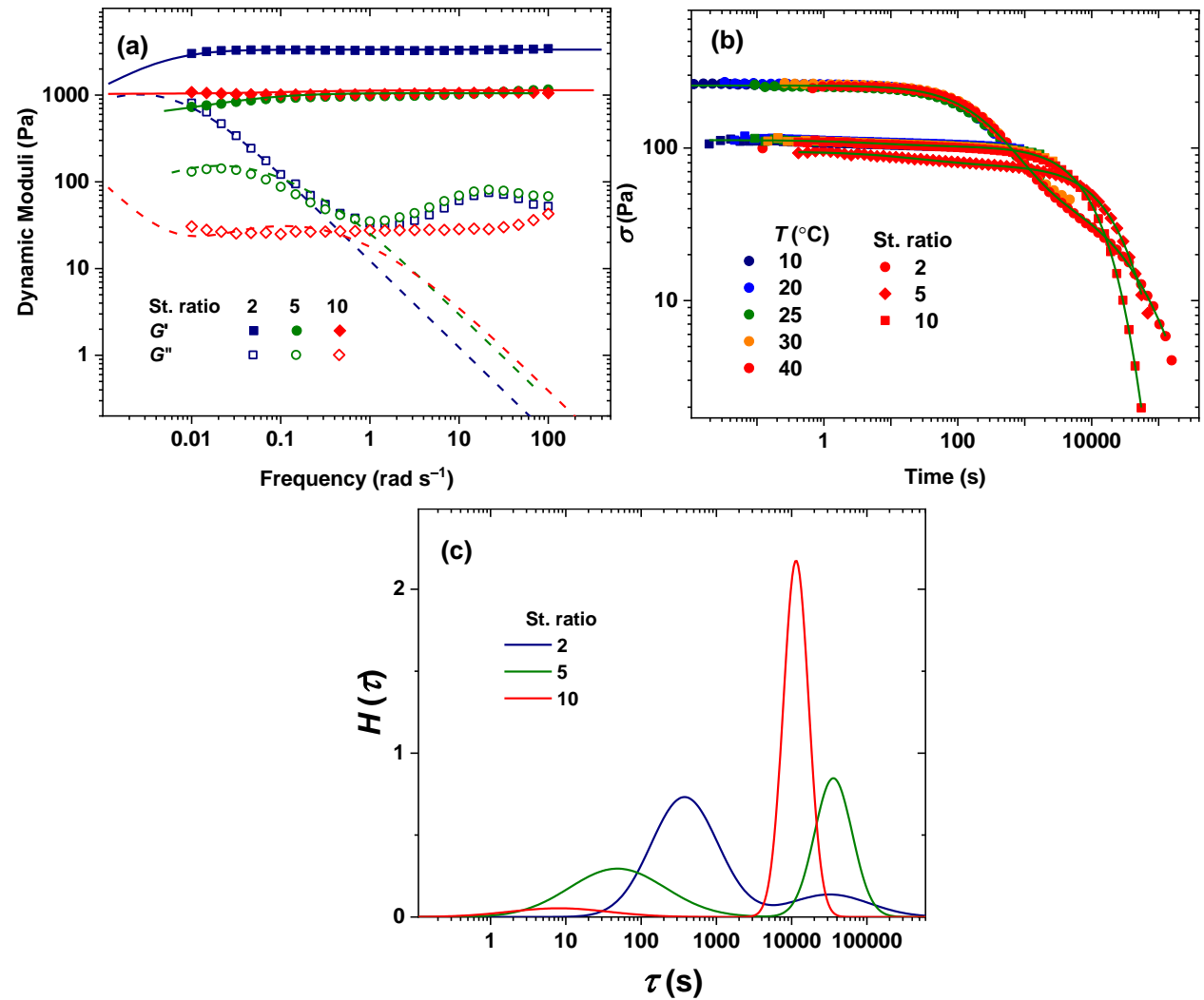

Figure 59. (a) Dynamic storage (filled symbol and solid lines) and loss (open symbols and dashed lines) moduli curves, (b) shear stress master-curves of transient networks at the reference temperatures of $25{ }^{\circ} \mathrm{C}$, and (c) relaxation time spectra of transient networks at the reference temperatures of $25{ }^{\circ} \mathrm{C}$, obtained by tetraMix $(0.5) \mathrm{Fe}(\mathrm{X})$ at $\varphi=60 \mathrm{~g} \mathrm{~L}^{-1}$ and metal to ligand stoichiometric ratios designated in legends. Solid shear stress lines are fitted curves obtained using up to three relaxation modes. Dynamic moduli lines are accordingly obtained by transforming step-strain fits to dynamic moduli. 